Revue d'histoire de l'Amérique française

19. REVUE D.HISTOIRE DE L'AMÉRIQUE FRANÇAISE

\title{
Lettre de Toussaint Rochon à sa femme
}

\section{Claude Perrault}

Volume 22, numéro 1, juin 1968

URI : https://id.erudit.org/iderudit/302754ar

DOI : https://doi.org/10.7202/302754ar

Aller au sommaire du numéro

Éditeur(s)

Institut d'histoire de l'Amérique française

ISSN

0035-2357 (imprimé)

1492-1383 (numérique)

Découvrir la revue

Citer ce document

Perrault, C. (1968). Lettre de Toussaint Rochon à sa femme. Revue d'histoire de l'Amérique française, 22(1), 89-92. https://doi.org/10.7202/302754ar d'utilisation que vous pouvez consulter en ligne.

https://apropos.erudit.org/fr/usagers/politique-dutilisation/ 


\section{DOCUMENTS INÉDITS}

\section{$-\mathrm{I}$ - \\ Lettre de Toussaint Rochon à sa femme}

Le 25 septembre 1839, à trois heures de l'après-midi, cinquante-huit prisonniers politiques du Bas-Canada apprennent, dans leurs cellules de Montréal, qu'ils seront déportés dans la NouvelleGalle-du-Sud [Australie]. Le départ est fixé pour le lendemain.

La cavalerie les escorte, menottés et liés deux à deux, jusqu'au pied du courant, au quai "Jilbort" 1 [Gilbert] où le vapeur British America les attend. Rendus à Québec, les déportés passent, le 27, à midi, sur le Buffalo, navire de guerre armé d'une vingtaine de canons, sous les ordres du capitaine Wood.

L'auteur de la lettre ci-dessous, Toussaint Rochon, forgeron et carrossier de Beauharnois, est l'un de ces exilés. A vingt-huit ans, il laisse une femme et deux enfants sans abri, à la suite de l'incendie de sa maison par les loyalistes. Parmi ses compagnons d'infortune, il compte deux de ses frères: Jérémie et PascalEdouard.

Grâce à l'amabilité de M. Georges-Henri Rochon, décédé récemment, nous avons pu prendre connaissance de nombreuses lettres des trois exilés Rochon. Celles-ci jettent un jour nouveau sur l'existence des déportés.

\section{Claude Perrault}

Long Bottom District de Paramanta Nouvelle galle Méridionale à Huit milles de Sydney dans la nouvelle Hollande, Cher Epouse

Apprès être rendu au lieux de ma destinations il me reste encore un sentiment de reconnoissance et datachement pour toi, et pour ma famille. Cher Sophie tu vouderoit avoir des lettres de moi, et Bien cher épouse en voilà une, que je t'écrit, et je seroit heureux si tu pouvait la recevoir comme je le désire, je t'écrit ce

$\overline{1 \mathrm{D}}$ 'après le journal de F.-M. Lepailleur, un autre déporté, dont nous avons copie du manuscrit. 
peut de mots pour te consolé dans tes peines, et afflictions, et pour t'aprendre Létât de ma santé, qui est bonne, j'ai été un peut malade durant la traverce, mais depuis que je suit à terre je jouit ainssi que mais compagnons d'unfortune de la meilleure sante [que] l'on puisse désirez. Cher Sophie je tavoit promit de técrire souvent et je ne l'ai point fait faute d'occations favorable en outre nous n'avons pas le droit de le faire qu'and nous le voulons. le capitaine du vesseaux nous à permit décrire, mais ne voulant pas t'aprendre que ma santé nétoit pas bien bonne, ni ne voulant te déguisé la vérité j'ai préféré ne pas écrire, j'avoit proposé d'écrire au cap Bonne espérance: mais le vent étant favorable nous en avons profité, nous sommes arrivé dans le port de Sydney le 25 Février nous avons resté 14 jours Sans débarquer un peut plus que le Gouverneur dici nous renvoya à l'Ils Norford qui est à 800 milles dici, et qui est une bien petite Ils, qui n'a que 20 milles de sirconférance si notre bon capitaine n'ut pas travailler pour nous auprès de son excellance, et des autres autorité nous y serions allez, car il paroit que le Gouverneur vouloit nous y envoyés ainsi que les autorité. Cher femme nous n'avons que des louanges à faire du capitaine du Buffalot tant pour la bonne conduite qu'il a husé envers nous que pour le bon caractère qu'il nous à donné avec autorités de ce lieux, qui nous à fait beaucoup de bien ance moment, si tu veut il n'a fait que ce qu'il étoit obligé de faire, car nous avons fait tous ce que nous devions faire pour leurs plaire, et faire notre devoir nous avons bien été traité dans le vesseaux autant que l'on peut le désirez dans un pareil cas. Le Buffalos est un vesseaux bien sure l'équipage bien prudente nous nous sommes rendu sans aucune accident qu'elconque et même sans avoir peur une seul fois, nous espérons tous nous en retourné si Dieu nous donne la liberté sous peut, Ses sous cette protections, et sur celle de la $\mathrm{S}^{\text {te }}$ Vierge que nous nous mettons et ces en eux que nous espérons toutes consolations, et toutes les grâces, si ce n'est pas en ce monde ce sera en lautre, une preuve que Dieu nous protège c'est que trois jours àprès que nous avons été arrivé dans le port, nous avons eût la visite de mon Seigneur l'évêque et de messire Labbé Brandy, prêtre, qui nous à fait un grand plaisir, car il y avait longtems que nous désirions avoir des prêtres, et le lendemain il est revenu (dire une basse messe) ${ }^{1}$ avec deux autres prêtres (et) ${ }^{2}$ nous confessé, et le sous lendemin il est revenu dire une base messe auqu'el il y à eu 51 qui ont communier et j'ai eu le bonneur d'être du nombre ainssi que mes frêres et tous les gens de Beauharnois, nous avons

1 Mots, entre parenthèses, rayés dans le texte.

2 Ibid. 
eus leurs visite depuis, et dans le temps de Pâque nous avons eu le même Bonneur et tous y ont participés, ce Bonneur nous à tous ranimé et fait prendre courage, nous avions désirez cette faveur et Dieu nous là accordé avant de mettre le pieds à terre. Cher Sophie nous sommes débarqué le 11 Mars pour aller rester à une établiscement que le gouvernement possède entre Sydney et Paramanta cette établissement se nomme Long Bottom, ce qui veut dire en françois Long fond, c'est une bien belle place, nous demeurons tous ensembles, et nous travaillons au compte du gouvernement, chacun à son occupation. Les uns case des petites pierre, les autres charie, et d'autre travaille de leurs metiers, pour moi j'ai préféré charier pour allez dans le chemin, mais nous espérons notre délivrance sous peut, car tous le monde sintéresse pour nous d'àprès ce que l'ont nous dit, nous somme conduit par un gentil homme qui est bien doux, et qui nous paroît être bien contant de nous chacun se conduit d'une manière irréprochable, nous espérons d'avoir notre liberté d'aller à l'Eglise avant d'avoir notre liberté dans l'Ils. Rien de plus à taprendre pour le présent pour l'ennui je n'ai pas besoin de tenparlé tu peut penser quelle doit être grande, étant séparé de ce que j'ai deplus cher au monde voir une femme dans la misaire et des enfans abandonné. Sans aucun (que le suport d'une) ${ }^{3}$ suport, que la protection d'une mère faible et désolé, qui n'a que la Providence pour espoir, pauvres enfants c'est moi qui suit lauteur de votre malheur, c'est moi qui deveroit tous souffrir non pas vous autres, puisse le ciel ramener au sain de sa famille des pères aussi malheureux que leurs sort les à rendu. Si jamais Dieu nous favorise d'un tel bonneur que de grâce aurons nous pas à lui rendre, la seul consolations qui nous reste, c'est de nous unir dans nos communions, et de prier Dieu qu'il daigne dans sa miséricorde nous réunir ensemble, je te prit cher femme si le malheur nous en veut délever nos enfans dans la crainte de Dieu de leur fair prier Dieu pour leur père toute affliger de leurs malheur, pour moi je ne vous oubli pas dans mes prières du matin et du soir; toujours je prirez Dieu pour vous car c'est la seul chose que je puisse vous faire dans ma malheureuse exil, je seroit heureux si je pouvoit vous exprimez ce que je ressent dans mon cœur. je termine enfain cher femme te priant d'accepter mes plus tendre adieu, aissi qu'à mes pauvres enfans Pauvres Orphelins il n'auront pas connu les douceur d'un père si le Ciel ne nous favorise. ... Embrasse ton père, et ta mère, tes frères, et sœurs, pour moi, fait mes (nos) ${ }^{4}$ complimens à nos parens et amis, mes frêres se joyent à moi pour tenbrassé de tous notre cœur, adieu cher femme ne cesse pas de prier Dieu pour moi.

3 Ibid.

4 Ibid. 
Joseph ton frêre, et André Papineau Se réunise à moi pour la même lettre que tu fera voir à leurs femmes, car je n'ai pas le tems de leurs en écrire, pour le présent, et celle ci doit sufir pour tous, le tems presse, adieu nos cher femmes adieu et nous tacherons de vous récrire et faites en autant si vous le pouvez; adieu chef femme et enfant à Dieu, à Dieu, à Dieu.

Ton marie pour la vie

Sydney Diman-

Toussaint Rochon

che le 17 Mai 1840

Reçu le 11 Novembre au midi 1840

${ }^{3}$ Mots rayés dans le texte. 